Courrier du Centre international Blaise Pascal

$11 \mid 1989$

Varia

\title{
Nouvelles pascaliennes
}

\section{Dominique Descotes}

\section{OpenEdition \\ Journals}

Édition électronique

URL : http://journals.openedition.org/ccibp/649

DOI : $10.4000 /$ ccibp. 649

ISSN : 2493-7460

\section{Éditeur}

Centre international Blaise Pascal

\section{Édition imprimée}

Date de publication : 20 décembre 1989

Pagination : $34-36$

ISSN : 0249-6674

\section{Référence électronique}

Dominique Descotes, « Nouvelles pascaliennes », Courrier du Centre international Blaise Pascal [En ligne], 11 | 1989, mis en ligne le 11 décembre 2015, consulté le 01 mai 2019. URL : http:// journals.openedition.org/ccibp/649; DOI : 10.4000/ccibp.649

Ce document a été généré automatiquement le 1 mai 2019.

Centre international Blaise Pascal 


\title{
Nouvelles pascaliennes
}

\author{
Dominique Descotes
}

\section{Publications}

1 Jansenistica Lovaniensia, revue du Centrum voor de Studie van het Jansenisme, a publié à la mémoire d'Émile Jacques, une plaquette intitulée Renseignements biographiques sur Émile Jacques; elle contient une brève autobiographie de M. Jacques et un article de Mathijs Lamberigts, «Émile Jacques et le Centrum voor de Studie van het Jansenisme » (Bibliothèque de la Faculté de Théologie, St Michielstraat 2-6, B 3000 Leuven, Belgique).

2 Le même centre vient de publier un Lexicon Pseudonymorum Jansenisticorum, répertoire de noms d'emprunt employés au cours de l'histoire du jansénisme et de l'antijansénisme, composé par J. Tans, E. Jacques, H. Schmitz du Moulin et M. Lamberigts. (Prix : 800 francs belges, frais d'envoi non compris ; même adresse que ci-dessus.)

3 M. Alain Vircondelet a publié à la librairie Flammarion Le Roman de Jacqueline et Blaise Pascal. La nuit de Feu (1989, 324 p.).

\section{Thèse}

4 Monsieur Hyung-Kil Kim a soutenu le 14 septembre 1989, à l'Université de Provence AixMarseille I, sous la direction de M. le Professeur Roger Duchêne, une thèse intitulée De l'Art de persuader dans les Pensées de Pascal. Le jury était composé de M. Roger Duchêne, M. Philippe Sellier, M. Antony McKenna et de M. Pierre Ronzeaud.

\section{Deuxième Prix Blaise Pascal}

5 La Ville de Clermont et le Club des Ouvreurs ont décerné le deuxième prix Blaise Pascal, d'un montant de 100000 francs, à M. Rémy Chauvin, pour son livre Dieu des Fourmis, Dieu des Étoiles (Édition du Pré aux Clercs). La remise du prix a eu lieu à l'Hôtel de Ville de 
Clermont-Ferrand, le 19 juin 1989, en présence de M. Roger Quilliot, Sénateur Maire. Le CIBP a été étroitement associé à la cérémonie. Une brochure, largement diffusée, conserve le souvenir de ce second prix.

\section{La Pascaline en carte postale}

Le Musée du Ranquet de Clermont-Ferrand propose de nouveau à ses visiteurs des cartes postales représentant la pascaline. Le précédent tirage, sur fond rouge, se trouvait complètement épuisé. Deux photographies sont désormais en vente (pour le prix d'un franc pièce), représentant l'une la machine décimale à huit roues, l'autre la machine du chevalier Durant-Pascal.

\section{BIBLIOGRAPHIE}

\section{Liste des ouvrages en dépôt au CIBP}

ERNST, Pol. - Approches pascaliennes. -Gembloux, 1970. - 709 p.

Blaise Pascal « Auvergnat ».Catalogue d'exposition - Clermont-Ferrand, 1981.

« Deux grandes figures d'Auvergne : Gilberte et Jacqueline Pascal ». Chroniques de Port-Royal -

Clermont-Ferrand, 1982.76 p. ill.

MOURLEVAT Guy. - Les Machines arithmétiques de Blaise Pascal. - Clermont-Ferrand, 1988. - 76 p., 52 p. de planches.

Jeu de quatre cartes postales noir et blanc représentant les filigranes du manuscrit des

«Pensées » de Pascal.

«Ordre par dialogues ».Récital donné à la Faculté des Lettres de l'Université Clermont II le 14 mai 1985, à l'occasion des journées « L’Accès aux Pensées de Pascal ».- 1 cassette.

«La violence et la vérité » : les « Provinciales » de Blaise Pascal : Récital donné le 16 mai 1986 à la

Faculté des Lettres de Clermont-Ferrand. - 1 cassette.

\section{Liste des dons reçus par le CIBP}

AKAGI Shozo, «Pascal et le problème du vide », Studies in the foreign languages and literature, Osaka, 3 (1967), 4 (1968), 5 (1969).

ARNAULD Antoine, Réplique à l'Anatomie de Monsieur l'Évesque de La Vaur, 1645, 1 vol.

ARNAUD D'ANDILLY Robert, Lettres, Lyon, 1665, 1 vol.

BOSMANS H., « Sur l'œuvre mathématique de Blaise Pascal », Revue des questions scientifiques, janv.-avr. 1924, vol. 85, p. 130-161 et 424-451. 
BRIMO Albert, Pascal et le droit, Toulouse, 1942 (thèse).

CHESTOV Léon, La nuit de Gethsémani : essai sur la philosophie de Pascal, Paris, 1923, 161 p.

DELACOUR André, Pascal et notre temps, Paris, 1923, 153 p.

DESCOTES Dominique, Aspects de la dialectique dans les Pensées de Pascal, Paris, 1970 (mémoire de maîtrise).

DESCOTES Dominique, « Les aspects littéraires et rhétoriques des avertissements dans les Lettres de A. Dettonville de Pascal ", La Marge, actes du colloque, Clermont-Ferrand, 1978, p. 109-132.

DROZDOWICZ Zbigniew, Intelectualizm i naturalizm wfilozofii Fracuskiej, Poznam, 1987, 47, 164 p.

FILLEAU DE LA CHAISE Jean, Discours sur les Pensées de monsieur Pascal, Lyon, 1694, 1 vol.

Fontenelle, actes du colloque (Rouen, 6-10 octobre 1987), Paris, 1989, 710 p.

FORCE Pierre, Le sens d'un auteur : étude du problème herméneutique chez Pascal, Paris, 1987, 382 f. (thèse).

FOURCADE Pierrette, Le baroque dans les fragments pour «l'Apologie », Toulouse, 1968 (thèse).

GAILLARD Abbé Jean, Discours prononcé à l'occasion de la distribution des prix à l'école Massillon, 27 juin 1962 (année du Tricentenaire de la mort de Pascal), Clermont Ferrand, 1962, 11p.

GIRBAL François, L'affaire du P. André Martin à Saumur (1669-1675), Paris, 1988, 129 p.

HALLAYS André, Les solitaires de Port-Royal, Paris, 1927, 122p.

HONG Ran-E, « La force et ses aspects chez Pascal », Équinoxe, Kyoto, 3 (1988), p. 508-526.

L'intelligence du passé..., Mélanges offerts à Jean Lafond par ses amis, Tours, 1988, 554 p.

ITARD Jean, Essais d'histoire des mathématiques..., Paris, 1984, 386 p.

JACQUES Émile, Jansénisme anti-jansénisme, acteurs, auteurs, témoins, Bruxelles, 1988, 1vol.

JOVY Ernest, Pascal n'a pas inventé le haquet : essai lexicographique, Paris, 1923, 14 p.

KIM Hyung-Kil, De l'art de persuader dans les Pensées de Pascal, Aix-Marseille, 1989, 348 f. (thèse).

KOYANAGI Kimiyo, «Sur l'esprit scientifique de Pascal : réflexions sur le traité du vide », 1975, p. 919-936.

KOYANAGI Kimiyo, "Pascal et l'expérience du vide dans le vide ", Japanese studies in the history of sciences, $\mathrm{n}^{\circ} 17,1978$.

Lexicon pseudonymorum Jansenisticorum, répertoire de noms d'emprunt employés au cours de l'histoire du Jansénisme et de l'antijansénisme, composé par J.A.G. Tans, E. Jacques, H. Schmitz du Moulin et M. Lamberigts, Leuven, 1989, 224 p.

«Livres hérétiques en la possession de Cornélius Jansénius », Jansenistica Lovaniensia, hiver 1987, $23 \mathrm{p}$.

LOCKWOOD Richard, "The reader's figure in Pascalian rhetoric », The Seventeeth century, Durharm, vol. IV, $\mathrm{n}^{\circ} 1$, Spring 1989, p. 67-76.

LORPHELIN, « Generatio rectorum benedicetur » (table généalogique de la famille Pascal), 1642 ca., $55 \times 128 \mathrm{~cm}$, double des ex. Rl7 et R18 de la BMIU Clermont-Ferrand.

MOROT-SIR Édouard, « Géométrie du sens, calcul de la parole : la raison selon Pascal », Semiotica, vol. $70^{2}, 1988$, p. 105-167.

MOURLEVAT Guy, Les machines arithmétiques de Blaise Pascal, Clermont-Ferrand, 1988, 76 p.+ 51 pl. 
Nouvelles littéraires, numéro spécial sur Blaise Pascal, 23juin 1923, 37 p.

OJARDIAS Albert, Le vrai Pascal et divers parmi les siens, Clermont-Ferrand, 1932, $32 \mathrm{p}$.

OHTOMO Hiroshi, Le problème du stoïcisme dans Pascal, Bordeaux, 1970 (thèse).

PASCAL Blaise, Le mystère de Jésus, composition et ornements dessinés et gravés sur bois par Louis Bouquet, Paris, 1918, $18 \mathrm{p}$.

PASCAL Blaise, CEuvres complètes, Paris, 1872-1877, 3 vol.

PASCAL Blaise, Pensées de Blaise Pascal, Paris, 1720, nouv. éd., 1 vol.

PASCAL Blaise, Pensées de Blaise Pascal, Paris, 1823, 2 vol.

PASCAL Blaise, Pensées, Paris, 1877, 1879, 2 vol.

PASCAL Blaise, Pensées, d'après l'éd. de M. Brunschvicg, Londres, Paris, 1913, 405 p.

PASCAL Blaise, Les Pensées, prés. par Marcel Guersant, Opuscules religieux et philosophiques, Paris, $1954,1090 \mathrm{p}$.

PASCAL Blaise, Pensées, t. 3, pub. par Yoichi Maeda, Tokyo, 1980, 211 p., photographies des fragments originaux et transcription littérale, trad., commentaire et notes en japonais, table de concordance, bibliographie ;

PASCAL Blaise, Les Provinciales, Cologne, 1689, 1 vol.

PASCAL Blaise, Prière à Dieu pour demander le bon usage des maladies, Paris, 1928, 84 p.

PASCAL Blaise, Les Provinciales ou lettres écrites par Louis de Montalte..., Cologne, 1699, 1 vol.

PASCAL Blaise, Les Provinciales, Clermont (en Auvergne), 1752, 1 vol.

Pascal, in Cahiers de l'Association internationale des études françaises, n 40, mai 1988, p. 199-329.

Pascal, thématique des Pensées, éd. par Lane M. Heller et Ian M. Richmond, Paris, 1988, 144 p.

RAFFELT Albert, «Pendent opera interrupta... », Freiburger Zeitschrift für philosophie und theologie, Freiburg, 35, 3 (1988), p. 508-526.

Recherches sur le XVII ${ }^{e}$ siècle, Centre d'histoire des sciences et des doctrines, Paris, 1986, 142 p.

RIBERY C., De infinito apud Pascalium, Insulae, Lille, 1903, 55 p.

SECHE Léon, « Un roman janséniste d'après les papiers de la famille de Barante », Revue de famille, décembre 1980, p. 445-464.

SELLIER Philippe, « Pour un Baudelaire et Pascal », p. 5-16.

SOELBERG Nils, « La dialectique de Pascal », Revue romane, 1978, t XIII, fasc. 2, p. 230-276.

SUARES André, Puissances de Pascal, Paris, 1923, 117 p.

SUEMATSU Hisashi, «Les Pensées et le métatexte, essai sur le symptôme de l'écriture inachevée », Équinoxe, n 1, automne 1987, p. 27-53.

TANABÉ Tamotsu, « Où fixer la règle ? Une clef pour déchiffrer le message pascalien », comment. en japonais, sommaire en français, 1984, p. 201-215.

TANNERY Paul, «Pascal et Lalouvère ", Mémoires de la société des sciences physiques et naturelles de Bordeaux, Paris, 1980 , t. IV, $4^{\circ}$ série et $\mathrm{t}$. $\mathrm{V}, 3^{\circ}$ série, $17 \mathrm{f}$. et $6 \mathrm{f}$.

UCHIDA Masao, «The feasibility of les expériences du vide dans le vide made by Pascal ", The bulletin of the faculty of humanities, Wako University, $n^{\circ} 17,1982$, p. 36-52 (ouvrage en japonais). 


\section{Achats effectués par le CIBP}

MAURRAS Charles, Pascal puni : conte infernal, Paris, 1953, 116 p.

PASCAL Blaise, Le Pari, avec un discours critique par Clément Besse, Paris, 1921, 69 p.

PASCAL Blaise, Lettres écrites à un provincial, Euvres de Pascal, Paris, 1886, 2 vol.

PASCAL Blaise, Les Provinciales, Cologne, 1657, 1 vol.

PASCAL Blaise, Texte primitif des lettres provinciales, Paris, 1867, XIX + 358 p.

SAINTE-BEUVE Charles Augustin, Port-Royal, Paris, 1926-1932, 10 vol.

SAINT-SIMON, Mémoires : corpus bibliographique, t. XXVI, Paris, 1988, 1134 p.

\section{Documents audio-visuels du CIBP}

MAEDA Yoichi : 2 photos.

Document Prix Blaise Pascal : 1 cassette vidéo, Mairie de Clermont-Ferrand.

La folie du Vide, 1648 : tirage photographique sur papier à partir du microfilm de l'exemplaire $\mathrm{R}$ 12377 de la Bibliothèque Nationale (en trois formats).

La folie du Vide, 1648 : microfilm de l'exemplaire R 12377 de la Bibliothèque Nationale.

PASCAL Blaise, Tombe de Blaise Pascal : épitaphe et stèle funéraire (2 photos sur papier en couleur).

Mélanges sur Pascal : Une bobine de microfilm, Ms 1186, Bibliothèque de la Sorbonne.

\section{AUTEUR}

DOMINIQUE DESCOTES

CERHAC, Université Blaise Pascal 